\title{
ORIGINAL ARTICLE SETD2 and histone H3 lysine 36 methylation deficiency in advanced systemic mastocytosis
}

\author{
G Martinelli ${ }^{1,20}$, M Mancini ${ }^{1,20}, \mathrm{C}$ De Benedittis ${ }^{1,21}, \mathrm{M}$ Rondoni $^{2,21}, \mathrm{C}$ Papayannidis ${ }^{1}, \mathrm{M}$ Manfrini ${ }^{1}$, M Meggendorfer ${ }^{3}, \mathrm{R} \mathrm{Calogero}^{4}$, \\ V Guadagnuolo ${ }^{1}$, MC Fontana ${ }^{1}$, L Bavaro ${ }^{1}$, A Padella $^{1}$, E Zago ${ }^{5,6}$, L Pagano ${ }^{7}$, R Zanotti ${ }^{8,9}$, L Scaffidi $^{8,9}$, G Specchia $^{10}$, F Albano ${ }^{10}$, \\ S Merante ${ }^{11}$, C Elena ${ }^{11}$, P Savini ${ }^{12}$, D Gangemi ${ }^{13}$, P Tosi $^{14}$, F Ciceri $^{15,16}$, G Poletti ${ }^{17}$, L Riccioni ${ }^{18}$, F Morigi ${ }^{18}$, M Delledonne $^{5,6}$, T Haferlach $^{3}$, \\ M Cavo ${ }^{1}$, P Valent $^{19}$ and S Soverini ${ }^{1}$
}

The molecular basis of advanced systemic mastocytosis (SM) is not fully understood and despite novel therapies the prognosis remains dismal. Exome sequencing of an index-patient with mast cell leukemia $(\mathrm{MCL})$ uncovered biallelic loss-of-function mutations in the SETD2 histone methyltransferase gene. Copy-neutral loss-of-heterozygosity at 3p21.3 (where SETD2 maps) was subsequently found in SM patients and prompted us to undertake an in-depth analysis of SETD2 copy number, mutation status, transcript expression and methylation levels, as well as functional studies in the HMC-1 cell line and in a validation cohort of 57 additional cases with SM, including MCL, aggressive SM and indolent SM. Reduced or no SETD2 protein expression-and consequently, H3K36 trimethylation-was found in all cases and inversely correlated with disease aggressiveness. Proteasome inhibition rescued SETD2 expression and H3K36 trimethylation and resulted in marked accumulation of ubiquitinated SETD2 in SETD2-deficient patients but not in patients with near-normal SETD2 expression. Bortezomib and, to a lesser extent, AZD1775 alone or in combination with midostaurin induced apoptosis and reduced clonogenic growth of HMC-1 cells and of neoplastic mast cells from advanced SM patients. Our findings may have implications for prognostication of SM patients and for the development of improved treatment approaches in advanced SM.

Leukemia (2018) 32, 139-148; doi:10.1038/leu.2017.183

\section{INTRODUCTION}

Systemic mastocytosis (SM) is characterized by abnormal growth and accumulation of mast cells (MCs) and their precursors in the bone marrow (BM) and in other organs. ${ }^{1}$ On the basis of clinical variables and signs of organ damage the WHO classification defines a spectrum of disease variants, including indolent SM (ISM), smoldering SM (SSM), SM with associated hematologic (non$\mathrm{MC}$ ) neoplasm (SM-AHN), aggressive SM (ASM) and MC leukemia (MCL). ${ }^{2-4}$ Clinical features and courses in patients with SM vary greatly. In those with ISM and SSM, the clinical course is usually stable and symptoms mostly relate to $M C$ degranulation and mediator release. ${ }^{1}$ In contrast, ASM and MCL are characterized by organ damage resulting from malignant $M C$ infiltration. Whereas patients with ISM are considered to have a normal life expectancy, ASM and MCL are severe, fatal forms of SM with short survival times. Therapeutic options are limited in these patients. Somatic mutations leading to ligand-independent activation of the receptor tyrosine kinase KIT (most frequently, KIT D816V) are considered to promote $\mathrm{MC}$ differentiation and survival and thus have an important pathogenetic role in SM. ${ }^{5}$ However, KIT D816V is detectable both in ASM and in most patients with ISM $^{6}$ and several lines of evidence suggest that KIT D816V alone is not a fully transforming oncoprotein. ${ }^{7,8}$ The investigation of a panel of candidate genes involved in myeloid neoplasms has recently shown that additional mutations in genes other than KIT (TET2, NRAS, ASXL1, DNMT3A, SRSF2, CBL, ASXL1) may indeed be found in advanced $S M$, including $S M-A H N$ and ASM. $^{9-11}$ Patients with additional mutations in ASXL1, RUNX1 and SRSF2 were found to have a significantly shorter overall survival. ${ }^{12-15}$ However, only one study using genome-wide approaches to screen for novel molecular aberrations in advanced SM (ASM or MCL) has been published to date. ${ }^{16}$ This prompted us to undertake an integrated molecular genetic study of a $\mathrm{MCL}$ case who was referred to our center, with the aim to identify novel, potentially druggable lesions involved in the pathogenesis of advanced SM.

\footnotetext{
${ }^{1}$ Department of Experimental, Diagnostic and Specialty Medicine, Hematology/Oncology 'L. e A. Seràgnoli', University of Bologna, Bologna, Italy; ${ }^{2}$ Ospedale Santa Maria delle Croci, Ravenna, Italy; ${ }^{3}$ MLL Munich Leukemia Laboratory, Munich, Germany; ${ }^{4}$ Department of Molecular Biotechnology and Health Sciences, University of Turin, Turin, Italy; ${ }^{5}$ Personal Genomics, University of Verona, Verona, Italy; ${ }^{6}$ Department of Biotechnologies, University of Verona, Verona, Italy; ${ }^{7}$ Institute of Hematology, Catholic University S. Cuore, Rome, Italy; ${ }^{8}$ Section of Hematology, Department of Medicine, Azienda Ospedaliera Universitaria Integrata di Verona, Verona, Italy; ${ }^{9}$ Multidisciplinary Outpatients Clinics for Mastocytosis (GISM), Azienda Ospedaliera Universitaria Integrata Verona, Verona, Italy; ${ }^{10}$ Department of Emergency and Organ Transplantation, Hematology Section, University of Bari, Bari, Italy; ${ }^{11}$ Department of Oncology-Hematology, Hematology Unit, Fondazione IRCCS Policlinico San Matteo, Pavia, Italy; ${ }^{12}$ Department of Internal Medicine, Faenza Hospital, Faenza, Italy; ${ }^{13}$ Hematology Unit, Frosinone, Italy; ${ }^{14}$ Hematology Unit, Rimini, Italy; ${ }^{15}$ University Vita-Salute San Raffaele, Milan, Italy; ${ }^{16}$ IRCCS San Raffaele Scientific Institute, Milan, Italy; ${ }^{17}$ Department of Clinical Pathology, Centro Servizi Pievesestina, AUSL Romagna, Pievesestina di Cesena, Italy; ${ }^{18}$ Unit of Anatomic Pathology, 'M. Bufalini' Hospital, Cesena, Italy and ${ }^{19}$ Department of Medicine I, Division of Hematology and Hemostaseology and Ludwig Boltzmann Cluster Oncology, Medical University of Vienna, Vienna, Austria. Correspondence: Dr S Soverini, Hematology/Oncology 'L. e A. Seragnoli', S. Orsola-Malpighi Hospital, Via Massarenti 9,40138 Bologna, Italy.

E-mail: simona.soverini@unibo.it

${ }^{20}$ Co-first authors.

${ }^{21}$ Co-second authors.

Received 6 October 2016; revised 16 May 2017; accepted 26 May 2017; accepted article preview online 16 June 2017 ; advance online publication, 30 June 2017
} 


\section{MATERIALS AND METHODS}

Patient samples and cell lines

The index MCL case and 57 SM patients subsequently included in the validation cohort based on sample availability ( 23 patients with ISM (three with SM-AHN), three with SSM, 20 with ASM (three with ASM-AHN) and 11 with MCL (two with MCL-AHN)) were diagnosed and classified according to WHO criteria. $^{2-4}$ The clinical characteristics of each patient are shown in Supplementary Table 1. In the index case, CD117+ cells were isolated from BM mononuclear cells (MNCs) collected at the time of diagnosis by immunomagnetic cell separation (Miltenyi Biotech, Cologne, Germany) followed by RNA, DNA and protein extraction with the AllPrep DNA/RNA/ Protein Kit (Qiagen, Hilden, Germany). Germline DNA was obtained from saliva. In the validation cohort, DNA, RNA and proteins were obtained from CD117+ cells or MNCs from BM. In this cohort, patients with acute myeloid leukemia-type AHN were excluded. As a result, almost all CD117+ cells isolated from BM samples were CD34-/CD117+ MCs. Investigators performing the experiments were blinded with respect to the WHO subtype, clinical features and outcome of the patients. Sample and data collection were approved by the Institutional Review Boards of the S. Orsola-Malpighi Hospital (protocol 112/2014/U/Tess) and the participating institutions. All patients gave written informed consent. The mast cell line HMC-1, generated from a patient with $M C L$, was kindly provided by $\mathrm{Dr}$ Joseph Butterfield (Mayo Clinic, Rochester, MN, USA). The cell line has two subclones, HMC-1.1 harboring the V560G KIT mutation, and HMC-1.2 harboring both the V560G and the D816V KIT mutations. ${ }^{17,18}$ The control cohort included 95 healthy individuals with age ranging from 20 to 95 years.

\section{Whole-exome sequencing (WES) and single-nucleotide} polymorphism (SNP)-arrays

For the index case, paired-end DNA libraries were prepared from CD117+ cells and from saliva using the TruSeq DNA Sample preparation kit (Illumina, San Diego, CA, USA). Exon-coding sequences were captured using TruSeq Exome Enrichment Kit (Illumina) and sequenced on an HiSeq 1000 (Illumina). SNP-array analysis was performed in the index case and in 13 additional cases of the validation cohort ( 8 MCL, 4 ASM, 1 ISM) using the CytoScan HD array (Affymetrix, Santa Clara, CA, USA). Protocols and data analysis are described in the Supplementary Information.

\section{Loss-of-heterozygosity ( $\mathrm{LOH}$ ) analysis at $3 \mathrm{p} 21.3$}

Genotyping of nine single-nucleotide polymophisms with minor allele frequency $>40 \%$ in the minimal common region of LOH (rs11720139, rs2305634, rs2305635, rs1079276, rs2278963, rs6767907, rs4082155, rs6780013, rs1531875) was performed by direct sequencing as detailed in the Supplementary Information.

\section{SETD2 mutation screening}

SETD2 mutations identified by WES in the index case were validated by Sanger sequencing at the DNA and mRNA level using primers shown in the Supplementary Information. Screening of SETD2 coding and promoter sequences in the HMC-1 cell line and in the validation cohort was performed by Sanger sequencing or high throughput sequencing as described in the Supplementary Information.

Quantitative reverse transcription (RT)-PCR for SETD2 expression In the HMC-1 cell line and in the 57 samples of the validation cohort, SETD2 expression was assessed by quantitative RT-PCR. Total RNA (200 ng) was reverse transcribed to CDNA with the High-Capacity CDNA Reverse Transcription Kit (Thermo Fisher Scientific, Waltham, MA, USA). Assays were performed in triplicate on the ABI 7900HT system (Thermo Fisher Scientific) using pre-designed TaqMan Gene Expression Assays (Thermo Fisher Scientific) for SETD2 ( $\mathrm{Hs} 01014784 \mathrm{~m} 1)$ and TBP ( $\mathrm{Hs} 00427621 \mathrm{~m} 1)$ as control gene. SETD2 mRNA levels were quantified using the Comparative $\mathrm{Ct}$ method, using a pool of 10 healthy donors of various ages as calibrator.

\section{Methylation analysis of the SETD2 promoter}

Methylation status at the SETD2 promoter was assessed in the validation cohort using EpiTect Methyl II PCR Assays (EPHS110216-1A; Qiagen) on an ABI PRISM 7900HT, according to manufacturer's instructions. A methylation sensitive (EPHS115450-1A) and methylation dependent (EPHS115451-1A) control assay were also run for all the samples analyzed. A pool of 10 healthy donors of various ages served as a negative control.

\section{Drug treatments}

After 24 hours of exposure of HMC-1.1 and HMC-1.2 cells to $1 \mu \mathrm{M}$ AZD1775 (Selleckchem, Houston, TX, USA), $1 \mu \mathrm{m}$ midostaurin (Selleckchem), $10 \mathrm{~nm}$ bortezomib (Selleckchem), $1 \mu \mathrm{m}$ AZD1775+1 $\mu \mathrm{m}$ midostaurin and $10 \mathrm{~nm}$ bortezomib $+1 \mu \mathrm{m}$ midostaurin, apoptotic cell death was assessed by measuring the uptake of fluoresceinated Annexin $\mathrm{V}$ and propidium iodide (PI) (Roche Diagnostics, Mannheim, Germany). A FACsCantoll flow cytometer (Beckton Dickinson, Franklin, NJ, USA) set at $488 \mathrm{~nm}$ excitation and $530 \mathrm{~nm}$ wavelength bandpass filter for fluorescein detection or $580 \mathrm{~nm}$ for PI detection, and a dedicated software (DIVA software; Beckton Dickinson) were used. Drug cytotoxicity was evaluated in clonogenic assays. In HMC-1.1 and HMC-1.2, the reduction of colony (generated in $0.9 \%$ methylcellulose supplemented with $30 \%$ fetal calf serum) number in the presence of increasing sublethal doses of AZD1775 (0.025-0.1 $\mu \mathrm{M})$, midostaurin (0.025-0.1 $\mu \mathrm{m})$, AZD1775+midostaurin (0.025-0.1 $\mu \mathrm{m}$ each), bortezomib $(0.25-1 \mathrm{nM})$, bortezomib+midostaurin $(0.25-1 \mathrm{~nm})+(0.025-$ $0.1 \mu \mathrm{m}$ ) was assessed after 10 days of incubation at $37^{\circ} \mathrm{C}$ in a fully humidified atmosphere and 5\% $\mathrm{CO}_{2}$. AZD $1775(0.025-0.1 \mu \mathrm{M})$, midostaurin

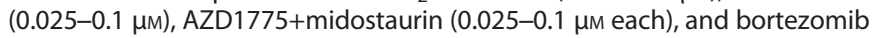
$(0.25-1 \mathrm{nM})$ were also tested in cells from three advanced SM patients (2 with MCL and one with ASM). Cells from two healthy donors were treated for comparison. Nonlinear regression analyses performed using the CompuSyn software ${ }^{19}$ (ComboSyn, Inc; Paramus, NJ, USA) were used to calculate the efficacy of different drugs alone or in combination in cell lines and in primary patient cells. Combination indexes were calculated using the same software.

\section{Co-immunoprecipitation/immunoblotting and western blotting analyses}

Co-immunoprecipitation/immunoblotting and western blotting were performed as described in the Supplementary Information using the following antibodies: anti-SETD2 (Abnova, Taipei, Taiwan), anti-H3K36Me3 (Merck Millipore, Darmstadt, Germany), anti-RNAPol II (Merck Millipore), anti-hnRNP (Merck Millipore), anti-SUMO (Cell Signaling Technology, Danvers, MA, USA) and anti-ubiquitin (Bethyl Laboratories, Montgomery, TX, USA). Beta-actin (Santa Cruz Biotechnology, Dallas, TX, USA) and Histone H3 (Merck Millipore) were used as loading controls. Immunoreactive proteins were visualized by probing with horseradish peroxidaseconjugated secondary antibodies and then by enhanced chemiluminescence (ECL; Thermo Fisher Scientific). Immunoblots were scanned and band intensities were quantified with the ImageJ software. Signal intensities in single blots obtained from three individual experiments were averaged, normalized to those of the loading controls and expressed as a fraction of the intensities, set to 1 , obtained in a pool of 10 healthy donors of various ages used as a control.

\section{Immunohistochemistry (IHC)}

Immunohistochemistry $(\mathrm{IHC})$ was performed on sections from paraffinembedded trephine BM biopsies of selected SM cases. IHC Staining with anti-SETD2 antibody (HPA042451, Sigma-Aldrich, Milan, Italy; 1:30 dilution) was performed using the Ventana BenchMark Ultra automated IHC slide staining system (Ventana Medical Systems, Tucson, AZ, USA). Tissues from normal testis and from a testicular seminoma were used as external controls and to determine the antibody dilution giving the best staining results. Antibody reactivity was visualized with diaminobenzidine. Slides were counterstained with hematoxylin.

\section{Statistical analysis}

Analysis of variance followed by Tukey post-hoc test was applied in order to detect differences in SETD2 and H3K36Me3 expression between groups. Spearman correlation index was calculated between SETD2 and H3K36Me3 level and disease subtype. Differences in the distribution of continuous and categorical variables between SETD2 $>0.5$ and $<0.5$ subgroups were assessed by means of non-parametric tests-the Mann-Whitney U-test and the Fisher's exact test, respectively. There was no adjustment for multiple testing as all analyses were explorative. Survival analysis was considered from the date of diagnosis to the date of death or last contact. Survival curves were prepared by the Kaplan and Meier method and statistical significance of differences in survival was calculated by the log- 
rank test. Analyses were carried out by SPSS (IBM Corp, Chicago, IL, USA) and $\mathrm{R}^{20}$ All $P$-values were two-sided and those less than 0.05 were considered statistically significant.

\section{RESULTS}

WES of the index MCL case identifies loss of function mutations in SETD2

Among the mutated genes found in neoplastic MCs but not in saliva, SETD2 stood out among others because of its known role as a tumor suppressor and its involvement in various solid tumors and in acute leukemias. ${ }^{21,22}$ Notably, two distinct putatively inactivating heterozygous mutations were identified in the patient, a nonsense mutation in exon 15 (NM_014159:C.G6753T: p.Glu2234Ter) and a frameshift insertion in exon 20 (NM_014159: c.7595_7596insC:p.Gly2515ArgfsTer5) (Figures 1a and b). Cloning of a cDNA fragment encompassing both mutant residues showed that the mutations were biallelic. The first mutation was predicted to result in a truncated protein lacking 330 amino acids at the C-terminal, whereas the second mutation was predicted to result in a truncated protein lacking 46 amino acids at the C-terminal. Western blotting, however, showed that only the SETD2-truncated isoform resulting from the nonsense mutation was expressed in neoplastic MCs (Figure 1c). This truncated protein (tSETD2) lacked the highly conserved SRI domain (Figure 1b). The normal SETD2 protein is responsible for trimethylation of lysine 36 of histone H3 (H3K36Me3). ${ }^{23}$ Co-immunoprecipitation showed that tSETD2 lost the ability to bind the $\mathrm{H} 3$ histone and, most importantly, western blotting showed that H3K36Me3 was abrogated (Figure 1c). The SRI domain is also known to mediate SETD2 interaction with the phosphorylated C-terminal domain of the RNA polymerase II large subunit ${ }^{24-26}$ and with the heterogeneous nuclear ribonucleoprotein- $L_{1}^{27}$ thus coupling $\mathrm{H} 3 \mathrm{~K} 36 \mathrm{Me} 3$ with transcription and splicing. Co-immunoprecipitation confirmed that tSETD2 did not bind either protein (Figure 1c), further underpinning the loss of function nature of the SETD2 mutations found in the index case.

$\mathrm{LOH}$ at the SETD2 locus is detectable in SM patients

The SETD2 gene maps to chromosome 3p21, where allelic loss due to deletions or copy neutral (CN)-LOH has frequently been observed in kidney, lung, breast and many other cancer samples and cell lines. ${ }^{28}$ We thus examined the copy number status and searched for LOH at 3p21 in 13 cases of the validation cohort (eight MCL, four ASM, one ISM) for whom SNP-array data were available. $\mathrm{CN}-\mathrm{LOH}$ of a small region ranging from $613 \mathrm{~kb}$ to $2.02 \mathrm{Mb}$ at $3 \mathrm{p} 21.3$ was found in 10/13 cases ( $7 \mathrm{MCL}, 2$ ASM, 1 ISM). The minimal common region (MCR) of $\mathrm{LOH}$ included SETD2 and nine additional genes (CCDC12, NBEAL2, NRADDP, KIF9-AS1, KIF9, $K L H 18$, PTPN23, SCAP, ELP6) (Supplementary Figure 1). We thus extended the screen for $\mathrm{LOH}$ specifically at $3 p 21.3$ in the remaining patients of the validation cohort. Genotyping of nine SNPs with minor allele frequency $>40 \%$ in the MCR was suggestive of $\mathrm{LOH}$ in 17 additional cases. Thus, overall, LOH was a

NM_014159:c.7595_7596insC:p.Gly2515 ArgfsTer5

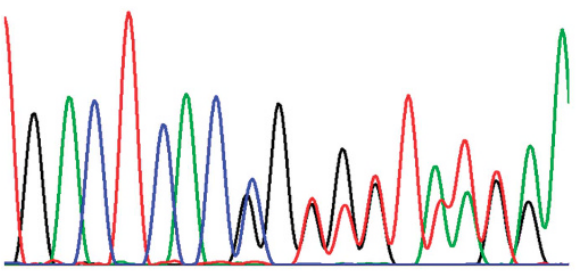

NM_014159:c.G6753T:p.Glu2234Ter

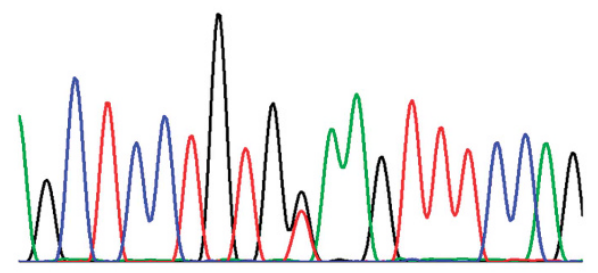

b

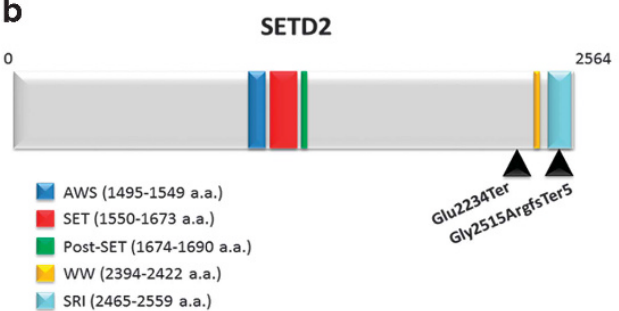

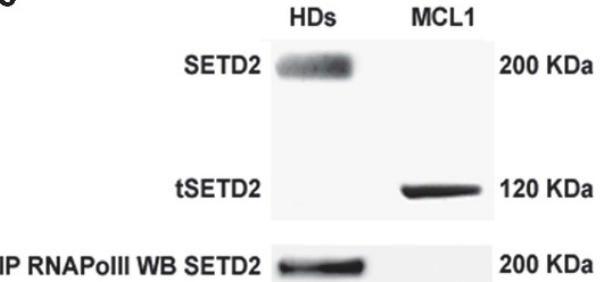

IP RNAPOIII WB SETD2 $200 \mathrm{KDa}$

$120 \mathrm{KDa}$
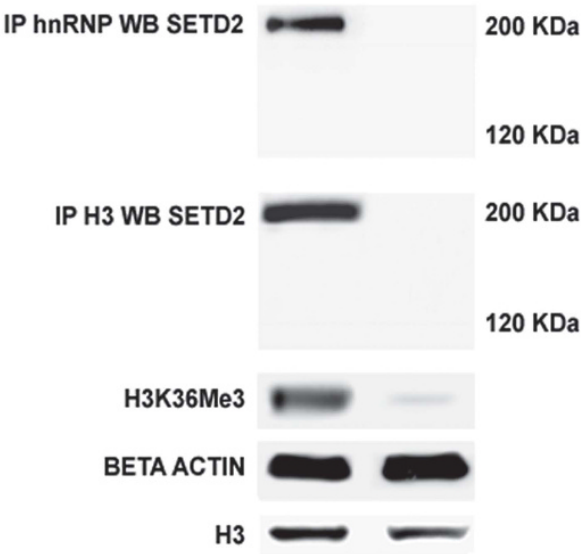

Figure 1. SETD2 loss of function mutations in the index MCL case (MCL1). (a) Sanger sequencing chromatograms with the frameshift (top) and nonsense (bottom) mutations identified by whole-exome sequencing. (b) Localization of the mutations with respect to the key functional domains of the SETD2 protein. The SRI domain is necessary for histone H3 lysine 36 trimethylation (H3K36Me3) and mediates SETD2 interaction with the phosphorylated C-terminal domain of the RNA polymerase II large subunit (RNA pol II) and with the heterogeneous nuclear ribonucleoprotein-L (HnRNP), thus coupling H3K36Me3 with transcription elongation and splicing. (c) from top to bottom: western blotting (WB) showing the truncated SETD2 (tSETD2) protein as compared to full-length SETD2 detectable in a pool of proteins from mononuclear cells of healthy donors; co-immunoprecipitation experiments performed by using: an anti-RNA pol II, an anti-hnRNP and an antihistone $\mathrm{H} 3$, respectively, to isolate the proteins of interest and then an anti-SETD2 as primary antibody to label the PVDF membrane on which the immunoprecipitates were transferred; WB for H3K36Me3. Histone $\mathrm{H} 3$ and actin were used as loading controls. 
detected in 27/57 (47\%) SM patients, namely in 8/11 (72\%) MCL-, $11 / 20$ (55\%) ASM-, 1/3 SSM- and 7/23 (30\%) ISM patients (Supplementary Table 2).

SETD2 and H3K36Me3 deficiency are recurrent events in SM and cluster in advanced disease

To investigate the frequency of SETD2 inactivation in the validation cohort, we used western blotting to assess H3K36Me3 levels as a surrogate marker of loss of SETD2 function and to screen for the expression of SETD2 full-length vs truncated isoforms. No evidence of abnormal isoforms was found. However, the SETD2 protein turned out to be reduced or not at all expressed in the great majority of patients examined (Figure 2a). H3K36Me3 paralleled SETD2 expression and was reduced or absent accordingly (Figure 2a). In one MCL, one ASM and two ISM patients, SETD2 and H3K36Me3 levels were assessed in total MNC fractions obtained from BM and in purified CD117+ cells in parallel and no differences were observed. To confirm western blotting results, IHC with an anti-SETD2 antibody was performed on BM trephine biopsies of patients displaying various SETD2 protein levels. The intensity of nuclear staining and the percentage of immunostained atypical MCs varied among the cases and were consistent with SETD2 protein expression as assessed by western blotting (Supplementary Figure 2). Internal positive controls (BM myeloid precursors and megakaryocytes) were present in each case and confirmed the specificity of the stain.

Median SETD2 and H3K36Me3 levels, assessed by densitometric analysis of western blotting results (Supplementary Table 3), were significantly correlated with each other (Spearman $R=0.91$, $P<0.001)$ and were lower in advanced SM $(R=0.57, P<0.001)$ (Figures $2 \mathrm{~b}$ and $\mathrm{c}$ ). In particular, patients with $\mathrm{MCL}$ and $\mathrm{ASM}$ displayed significantly lower levels of SETD2 and H3K36Me3 as compared to ISM (SETD2: $P=<0.001$ and $P=0.002$, respectively; H3K36Me3: $P \leqslant 0.001$ and $P=0.004$, respectively). There seemed to be no differences in SETD2 expression between advanced SM patients with $(n=8)$ or without $(n=26)$ AHN. When the distribution of SETD2 protein levels within the ASM and ISM subgroups was examined, no significant differences regarding baseline clinical variables or survival emerged between cases with SETD2 $<0.5$ and $>0.5$ (data not shown). However, when patients with SETD2 $>0.5$ and $<0.5$ were compared regardless of disease subtype, lower SETD2 expression was found to be associated with older age $(P=0.02)$, higher serum tryptase levels $(P=0.03)$, lower hemoglobin $(P=0.03)$ and platelet count $(P=0.005)$. In addition, patients with lower SETD2 levels were more likely to have splenomegaly $(P=0.04)$ and less likely to have skin lesions $(P=0.03)$. There was also a trend towards shorter overall survival $(P=0.06)$ (data not shown). There seemed to be no preferential association between SETD2 loss of function and the presence or absence of specific additional mutations or mutation patterns in myeloid genes (for example, SF3B1, SRSF2, TET2, ASXL1 and so on; Supplementary Table 4).

In an attempt to explain the nonrandom accumulation of $\mathrm{LOH}$ at 3 p21.3 and the observed SETD2 protein deficiency, we sequenced the entire SETD2 gene, including the promoter region, in all the patients of the validation cohort. Only one ASM patient (who had no evidence of 3p21.3 LOH) had a missense mutation

a

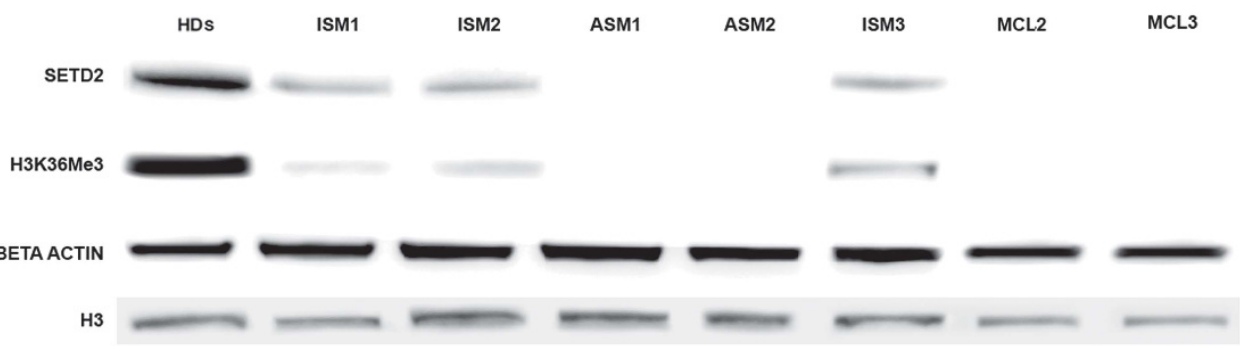

b

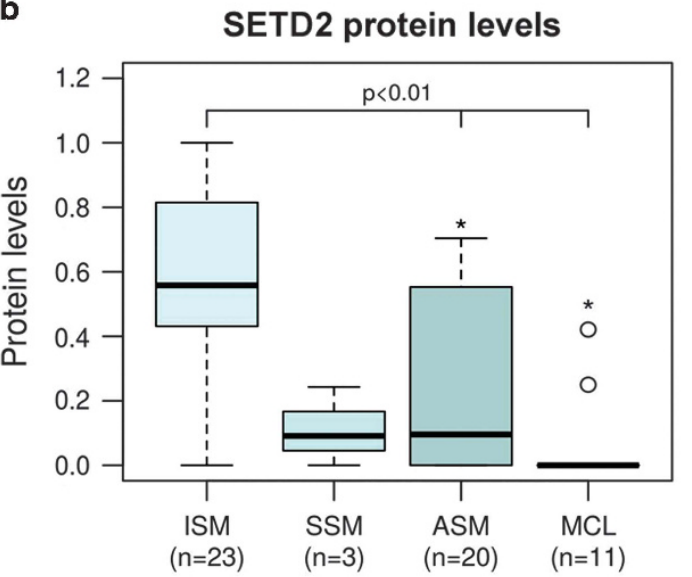

Groups
C

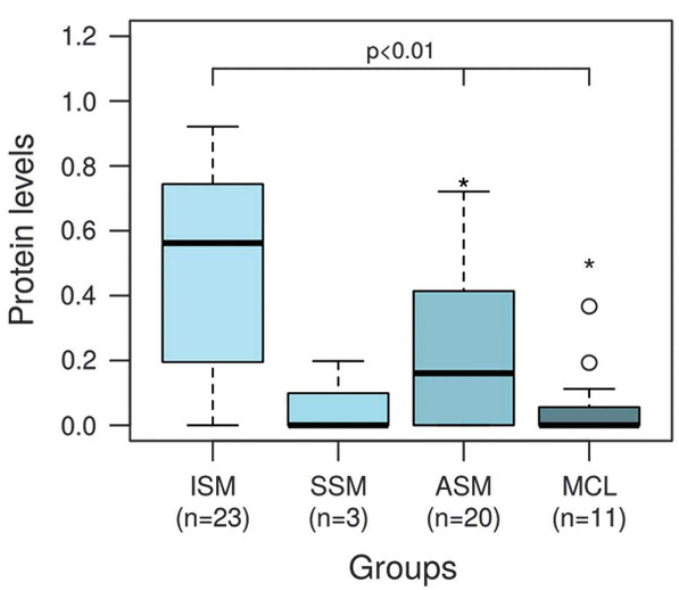

Figure 2. SETD2 protein and H3K36Me3 deficiency in SM. (a) representative western blot results for SETD2 protein and H3K36Me3 levels in SM patients as compared to a pool of healthy donors (HDs). One of three independent experiments is shown. (b and c) Box and whiskers plots of SETD2 and H3K36Me3 level estimates obtained by densitometric analysis of western blots. Median, interquartile range, minimum, maximum and outliers are indicated. SETD2 and $\mathrm{H} 3 \mathrm{~K} 36 \mathrm{Me} 3$ signal intensities in single blots obtained from three individual experiments were normalized to those of beta-actin and H3 histone, respectively, and averaged. Normalized SETD2 and H3K36Me3 levels calculated in SM patients were then expressed in comparison to normalized SETD2 and H3K36Me3 levels detected in a pool of HDs, conventionally set to 1 . The asterisks indicate that MCL and ASM had significantly lower levels of SETD2 protein $(P<0.001$ and $P=0.002$, respectively) and H3K36Me3 $(P<0.001$ and $P=0.004$, respectively) as compared to ISM. 
(c.1351A > G; p.Thr451Ala) not reported as a SNP (Supplementary Table 5). The mutation was not found either in the Catalogue of Somatic Mutatons in Cancer or in ClinVar databases and was predicted to be benign/tolerated by Mutation Taster and Polyphen-2. Thus, despite LOH, the classical two-hit hypothesis of tumor suppressor gene inactivation was found not to be the case for SETD2 in SM.

We then assessed whether promoter hypermethylation might be responsible for SETD2 protein deficiency, in the presence or absence of LOH. Analysis of the SETD2 promoter showed $<1 \%$ methylated DNA in all the patients of the validation cohort. Methylation levels were not different from those of healthy donors. Other mechanisms of transcriptional down-regulation were excluded, since SETD2 mRNA levels were not significantly lower in the validation cohort as compared to healthy donors.

To rule out the possibility that SETD2 loss of function might be another age-associated alteration in an epigenetic regulator, ${ }^{29-31}$ 80 healthy older adults with an age ranging from 65 to 95 years were screened for SETD2 mutations and for alterations in SETD2 protein expression or $\mathrm{H} 3 \mathrm{~K} 36 \mathrm{Me} 3$. No sequence variants other than the Pro1962Leu and the Asn1155Asn SNPs were detected. All the older adults did express SETD2; and densitometric analysis of western blots (done using a pool of 10 younger adults with an age ranging from 20 to 30 years as a calibrator, after having excluded inter-individual variations of expression) showed comparable SETD2 and H3K36Me3 levels among individual older adults as well as between older and younger adults (Supplementary Table 6).

We next wondered whether the HMC-1 cell line, established from a patient with $\mathrm{MCL}$, also exhibits SETD2 and H3K36Me3 deficiency. Indeed, western blotting revealed no SETD2 protein and no $\mathrm{H} 3 \mathrm{~K} 36 \mathrm{Me} 3$ in either subclone (Figure 3a). Sequencing ruled out the presence of SETD2 gene mutations. Similarly to the patients of the validation cohort, both HMC-1.1 and HMC-1.2 cells were found to have no significant reduction in SETD2 transcript levels as compared to SETD2 wild-type cell lines and the gene promoter was found to have very low methylation levels $(4.08 \%$ and $1.08 \%$ in HMC-1.1 and HMC-1.2, respectively). This suggested that similar mechanism(s) acting at the translational or posttranslational level underlie SETD2 deficiency in advanced SM patients and in HMC-1 cells.

SETD2 deficiency results from enhanced proteasomal degradation In HMC-1 cells, incubation with the proteasome inhibitor bortezomib (10 $\mathrm{nm}$ for $24 \mathrm{~h}$ ) rescued SETD2 protein expression and restored $\mathrm{H} 3 \mathrm{~K} 36 \mathrm{Me} 3$ levels (Figure $3 \mathrm{~b}$ ), indicating that a functional SETD2 protein is regularly translated in neoplastic MCs, and that failure to detect it by western blotting is rather due to altered turnover and proteasomal degradation. Coimmunoprecipitation with an anti-SETD2 antibody after bortezomib treatment showed that blockage of proteasome-mediated degradation results in accumulation of ubiquitinated and SUMOylated SETD2 (Figure 4a). We therefore speculated that reduced or undetectable SETD2 expression in advanced SM patients might be accounted for by hyper-ubiquitination and -SUMOylation leading to proteasomal degradation. We thus compared the effects of proteasome inhibition in malignant MCs from advanced SM patients displaying no SETD2 protein and from ISM patients with near-normal SETD2 levels. In contrast to SETD2-deficient patients, where results were superimposable to those obtained in HMC-1 cells, patients with near-normal SETD2 expression had similar levels of ubiquitinated and SUMOylated SETD2 protein (and similar H3K36Me3 levels) before and after the inhibition of proteasome-mediated degradation (Figure 4b).

Bortezomib and the combination of Midostaurin+AZD1775 reduce colony growth and induce apoptosis in HMC-1 cells

Given the ability of bortezomib to rescue SETD2 expression and H3K36Me3, we tested whether proteasome inhibition might be effective in vitro in HMC-1 cells. Bortezomib as single agent strikingly inhibited colony formation $\left(\mathrm{LD}_{50}\right.$ in $\mathrm{HMC}-1.1=0.173 \mathrm{nM}$; $\mathrm{LD}_{50}$ in HMC-1.2 $=0.226 \mathrm{~nm}$ )(Figure $5 \mathrm{a}$ ) and induced apoptosis in these cells (Figures $5 \mathrm{f}$ and $\mathrm{g}$ ). Combination with D816V KIT tyrosine kinase inhibition by midostaurin ${ }^{32,33}$ did not increase significantly bortezomib efficacy (Supplementary Figure 3).

Recently, a synthethic lethality interaction between H3K36Me3 deficiency and enhancement of replicative stress via Wee1 G2 checkpoint Serine/Threonine protein kinase inhibition has been uncovered. ${ }^{34}$ As a consequence, inhibition of Wee1 with AZD1775 was found to kill SETD2/H3K36Me3-deficient cells. ${ }^{34}$ We thus decided to explore also the in vitro efficacy of a strategy using AZD1775 alone or in combination with midostaurin in HMC-1 cells. AZD1775+midostaurin combination suppressed colony growth with a $\mathrm{LD}_{50}$ of $0.036 \mu \mathrm{m}$ and $0.038 \mu \mathrm{M}$ for HMC-1.1 and -1.2, respectively (Figure $5 \mathrm{~b}$; results of AZD1775 and midostaurin as single agents shown in Figures $5 \mathrm{c}$ and $d$; summary of LD $_{50}$ values in Figure 5e). When compared to either agent alone, AZD1775 +midostaurin combination displayed a strong synergistic effect in apoptotic cell death induction in HMC-1.1 cells, whereas only an additive effect was observed in HMC-1.2 cells (Supplementary Table 7).

Bortezomib and the combination of AZD1775+midostaurin reduce the clonogenic potential of SETD2/H3K36Me3-deficient neoplastic MCs

We next assessed the effects of bortezomib, AZD1775, midostaurin and AZD1775+midostaurin combination on the clonogenic potential of neoplastic MCs from two patients with MCL with no SETD2 expression (MCL3 and MCL7) and one patient with ASM (ASM18) with very low SETD2 expression (20\% as compared to a pool of healthy donors). Growth inhibition curves and LD $_{50}$ values

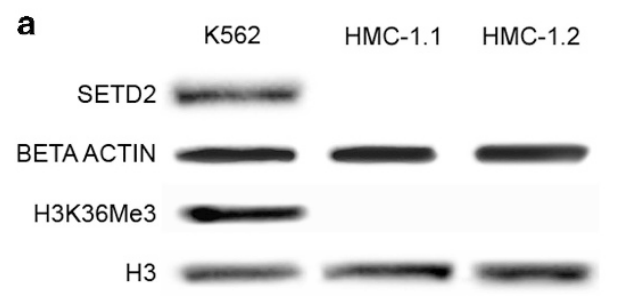

b

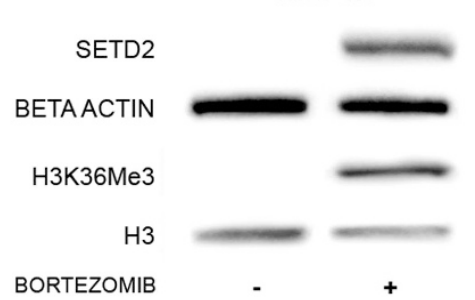

C

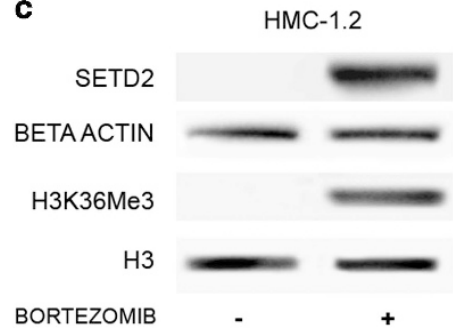

Figure 3. SETD2 protein and H3K36Me3 levels in HMC-1.1 and -1.2 cell lines before and after inhibition of proteasome-mediated degradation. (a) SETD2 and H3K36Me3 levels as detected by western blotting in HMC-1.1 and HMC-1.2 cells compared to K562 cells. (b and c) Effect of proteasome inhibition by bortezomib on SETD2 and H3K36Me3 levels in HMC-1.1 and HMC-1.2. One of three independent experiments is shown in all panels. 


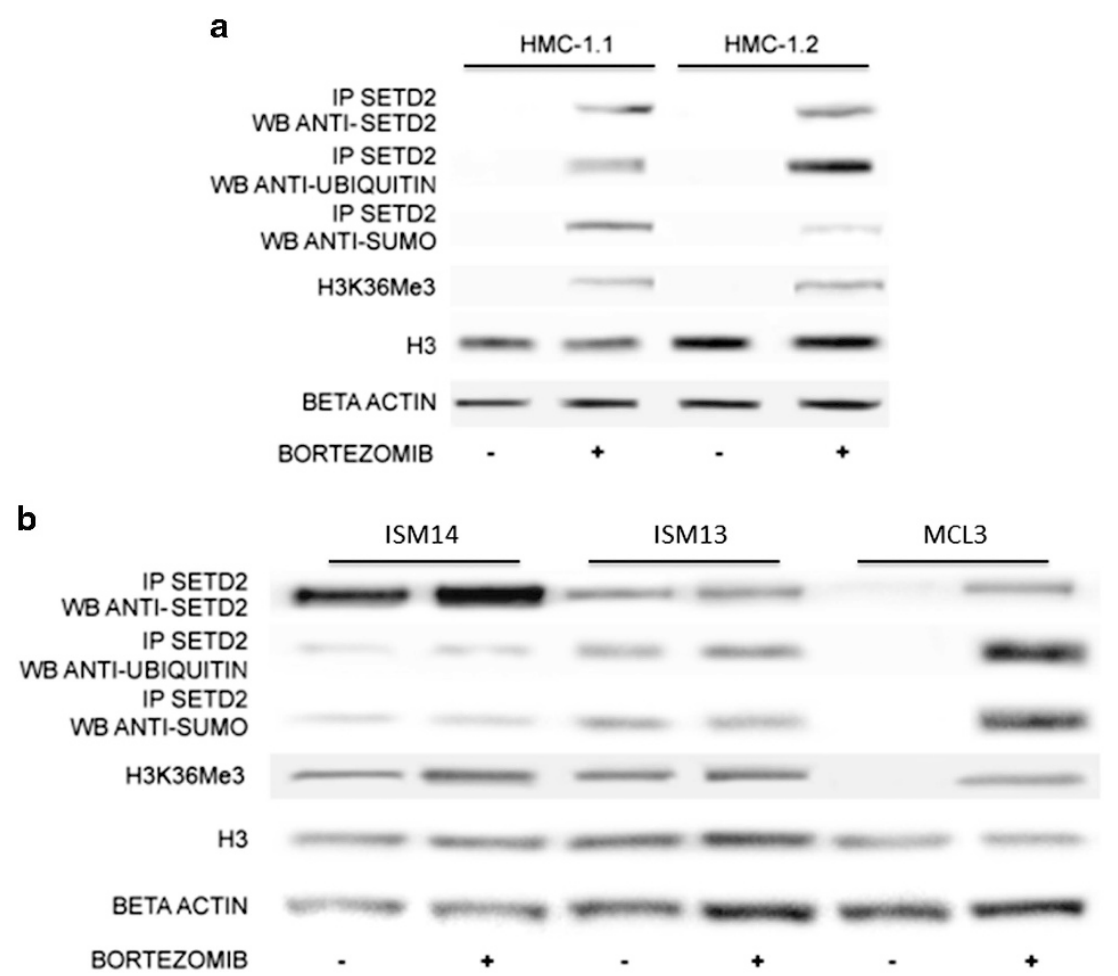

Figure 4. Ubiquitinated and SUMOylated SETD2 after proteasome inhibition by bortezomib in HMC-1 cells (a) and in SM patients (b). Co-immunoprecipitation (IP) with an anti-SETD2 antibody and immunoblotting (western blotting) with anti-ubiquitin and anti-SUMO antibodies performed before and after bortezomib treatment (10 $\mathrm{nm}$ for $24 \mathrm{~h}$ ). H3K36Me3 levels were also assessed by WB. H3 and beta-actin were used as controls. The two ISM patients had near-normal SETD2 protein levels $(90.5 \%$ and $75.8 \%$ of SETD2 expression with respect to a pool of healthy donors); the MCL patient had no SETD2 protein expression (see Supplementary Table 3 for SETD2 protein level estimates as assessed by densitometric analysis of western blots). One of three independent experiments is shown in all panels.

are shown in Figure 6. Bortezomib induced a marked dosedependent reduction in colony formation with $\mathrm{LD}_{50}$ ranging from 0.105 to $0.295 \mathrm{~nm}$ (Figure 6a). The combination of AZD1775 +midostaurin appeared to be slightly superior to the single agent strategy in MCL3 and ASM18, with LD $_{50}$ values of 0.054 and $0.058 \mu \mathrm{M}$, respectively, but not in MCL7 where AZD1775 as single agent showed the lowest $\operatorname{LD}_{50}(0.026 \mu \mathrm{m}$ for AZD1775 alone as against $0.048 \mu \mathrm{m}$ for the combination; Figures $6 \mathrm{~b}-\mathrm{e})$.

\section{DISCUSSION}

In recent years, deregulation of epigenetic modifiers by chromosomal translocations, genomic loss and/or point mutations has turned out to be a recurrent event in cancer evolution. Loss of function of the SETD2 tumor suppressor gene, which encodes the only histone methyltransferase that can catalyze H3K36Me3, was first identified in clear cell renal cell carcinomas, ${ }^{35}$ and subsequently, albeit at lower frequency, in several other solid tumors including lung, breast, skin and brain cancers. ${ }^{36}$ More recently, SETD2 loss of function and global loss of H3K36Me3 have been observed in acute lymphoid and myeloid leukemias. ${ }^{37,38}$ In renal cell carcinomas, SETD2 loss of function mainly results from large monoallelic deletions or CN-LOH at chromosome 3p, followed by mutation of the remaining allele. ${ }^{33}$ In acute leukemias, it rather derives from biallelic missense or truncating mutations. Here, we report for the first time that SETD2 and H3K36Me3 deficiency are recurrent events in patients with advanced SM. Of note, our findings demonstrate that SETD2 loss of function may also occur at the post-translational level, in the absence of mutations or structural aberrations. Biallelic inactivating mutations were indeed found only in the index case. $\mathrm{LOH}$ of a relatively small region at 3p21.3 was detected in almost 50\% of the patients of the validation cohort, with higher frequency in advanced SM as compared to ISM. However, LOH was neither associated with coding, splicing or promoter mutations, nor with epigenetic silencing, and no significant differences in SETD2 mRNA levels were found when comparing SM patients with or without LOH and healthy donors. Nonetheless, the great majority of the patients had reduced SETD2 protein expression, albeit to a varying degree, as compared to a pool of healthy donors as assessed by western blotting. As expected, this was mirrored by similarly reduced, or totally abrogated, H3K36Me3 (Figure 2a). IHC in BM biopsies of the same patients further confirmed this observation and showed weak or no nuclear staining for SETD2 in neoplastic MCs (Supplementary Figure 2).

Very low or absent SETD2 protein expression was especially found in cases with advanced SM (Figure 2b), with some exceptions. The most striking outliers were three ISM patients with no SETD2 expression by western blotting and IHC: one of these patients was a severely symptomatic ISM case, with histologically documented gastrointestinal involvement; one had diffuse osteoporosis; the remaining one seemed to have no peculiar clinical features. The small number of patients belonging to each disease subtype limited the possibility to perform statistical comparisons to identify peculiar clinical features or outcome differences between patients with high vs low SETD2 expression within the ASM and ISM groups. When patients with high and low SETD2 levels were compared irrespective of the WHO category, lower SETD2 levels correlated with older age, higher serum tryptase levels, lower hemoglobin and platelet count, splenomegaly and absence of skin lesions. There was also a not statistically significant trend towards shorter overall survival. In 

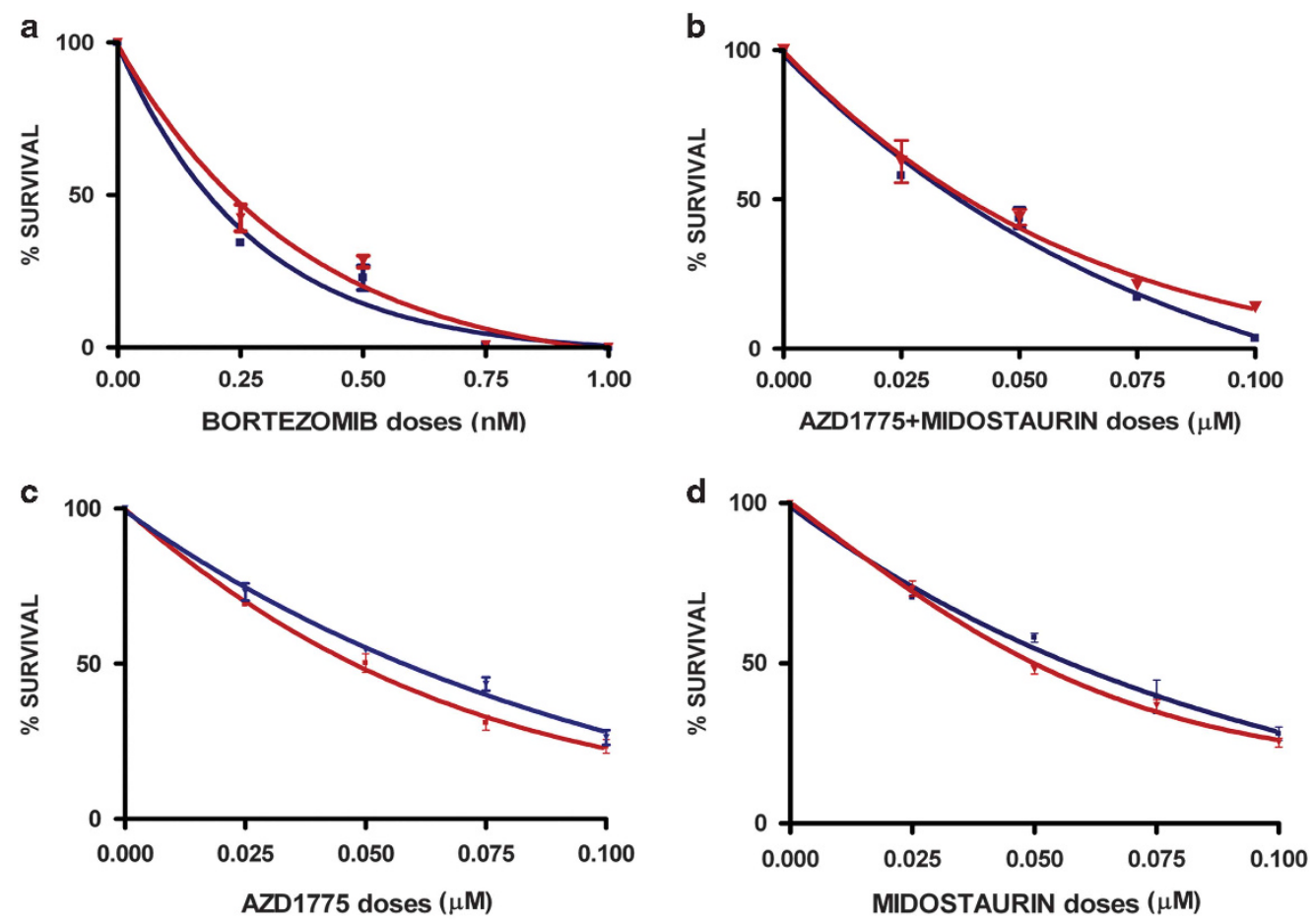

e

\begin{tabular}{|l|l|l|}
\hline DRUGS & HMC 1.1 & HMC 1.2 \\
\hline BORTEZOMIB & $0.173 \mathrm{nM}$ & $0.226 \mathrm{nM}$ \\
\hline AZD1775+MIDOSTAURIN & $0.036 \mu \mathrm{M}$ & $0.038 \mu \mathrm{M}$ \\
\hline AZD1775 & $0.058 \mu \mathrm{M}$ & $0.046 \mu \mathrm{M}$ \\
\hline MIDOSTAURIN & $0.057 \mu \mathrm{M}$ & $0.049 \mu \mathrm{M}$ \\
\hline
\end{tabular}
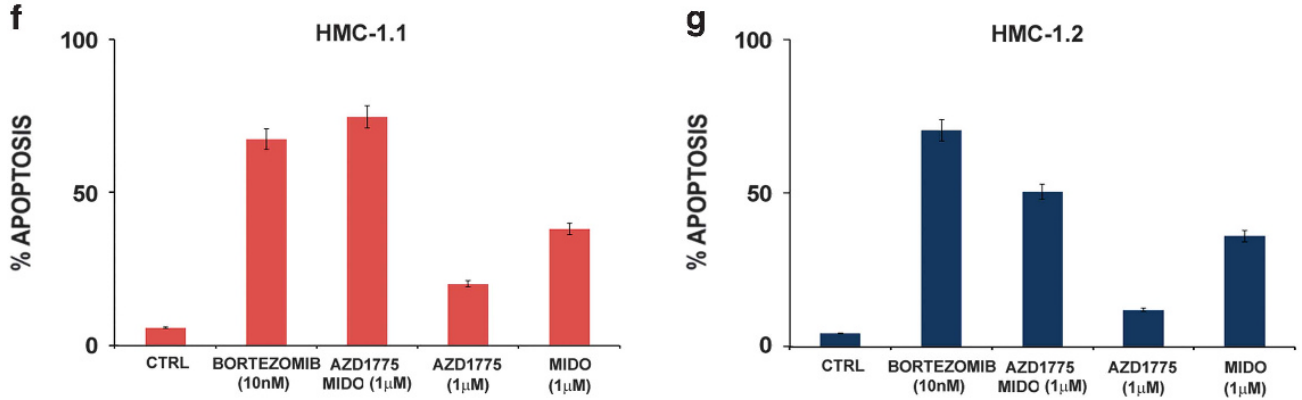

Figure 5. Effects of bortezomib and AZD1775+midostaurin combination in HMC-1 cells. (a and b) reduction of clonogenic growth of HMC-1.1 (red curve) and -1.2 (blue curve) cells, in the presence of increasing doses of bortezomib (0.25-1 nM) and AZD1775+midostaurin (0.025-0.1 $\mu \mathrm{m}$ ), respectively. All the clonogenic survival rates are expressed as mean \pm standard deviation of counts from three independent experiments. Curves with AZD1775 $(0.025-0.1 \mu \mathrm{m})$ and midostaurin $(0.025-0.1 \mu \mathrm{M})$ as single agents are shown in $\mathbf{c}$ and $\mathbf{d}$. (e) comparison of LD 50 values for the different strategies. (f and $\mathbf{g}$ ) Induction of apoptosis in HMC-1.1 and -1.2, respectively. Columns represent the mean of three independent experiments and the bars represent the standard error. ctrl, control; mido, midostaurin.

this regard it is noteworthy that in renal cell carcinomas lower SETD2/H3K36Me3 levels, assessed by IHC, correlate with larger tumor burden, more advanced stage of disease, and a worse prognosis. ${ }^{39,40}$ Similarly, in chronic lymphocytic leukemia recent data suggest that SETD2 monoallelic deletions or mutations are associated with inferior progression-free and overall survival. ${ }^{41}$ Analysis of a larger series of patients is warranted to fully understand the clinical and prognostic meaning of SETD2 loss of function in SM.

Proteasomal degradation was found to have a major role in the observed lack of SETD2 protein. In the HMC-1 cell line and in neoplastic MCs from advanced SM patients, incubation with the proteasome inhibitor bortezomib rescued SETD2 expression and H3K36Me3. Immunoprecipitation and immunoblotting showed that blockage of proteasomal degradation results in accumulation of ubiquitinated and SUMOylated SETD2 in HMC-1 cells and in primary MCs in advanced SM patients with no SETD2 protein expression but not in ISM patients with near-normal SETD2. This evidence of 'non-genomic' loss of function suggests that measuring protein and $\mathrm{H} 3 \mathrm{~K} 36 \mathrm{Me} 3$ levels by western blotting or $\mathrm{IHC}$ is the most informative screening approach and that the frequency of SETD2 involvement in solid tumors or hematologic malignancies might be higher than sequencing and copy number data alone would suggest. 

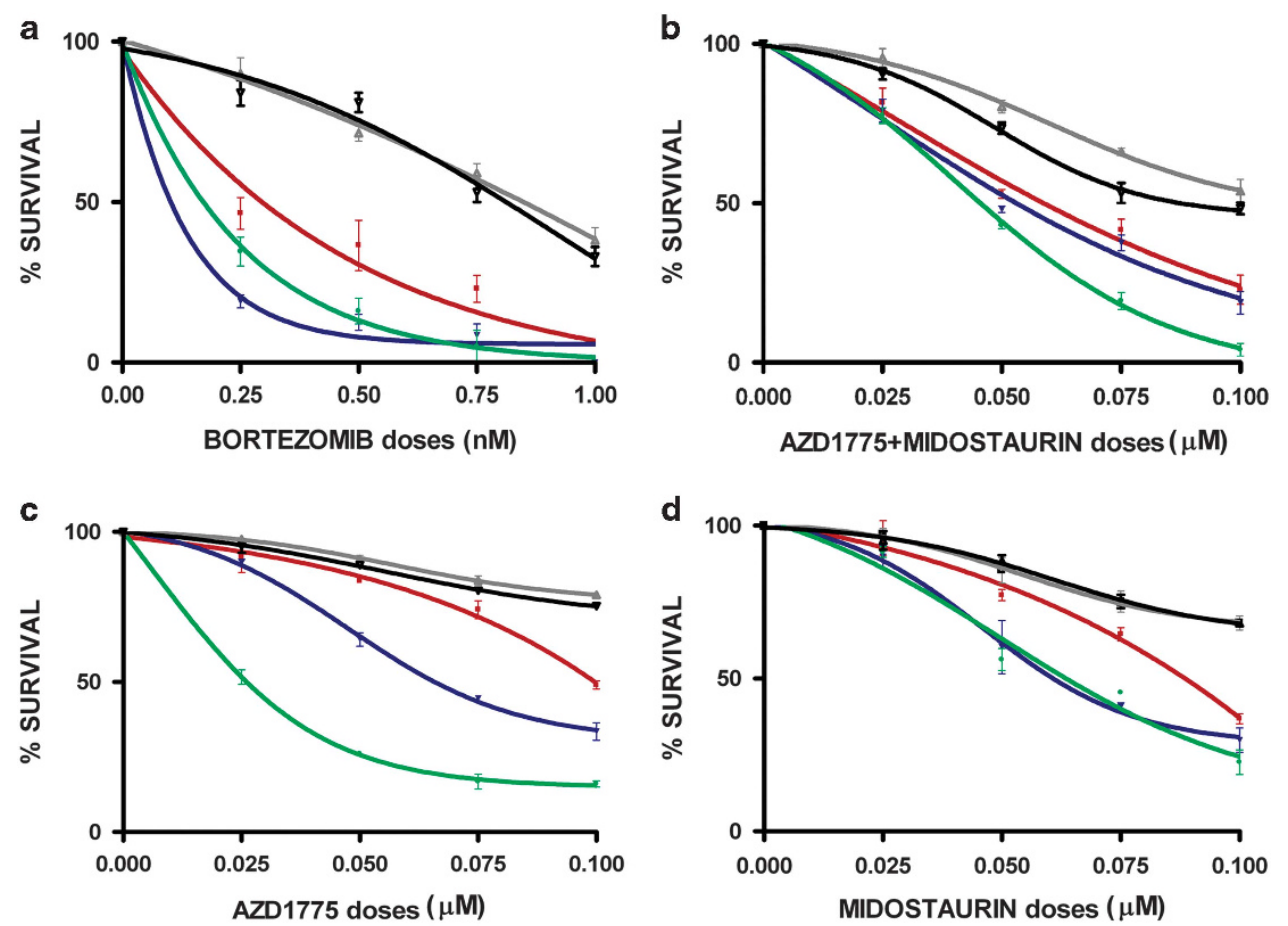

e

\begin{tabular}{|l|c|c|c|}
\hline DRUGS & MCL3 & MCL7 & ASM18 \\
\hline BORTEZOMIB & $0.105 \mathrm{nM}$ & $0.168 \mathrm{nM}$ & $0.295 \mathrm{nM}$ \\
\hline AZD1775+MIDOSTAURIN & $0.054 \mu \mathrm{M}$ & $0.048 \mu \mathrm{M}$ & $0.058 \mu \mathrm{M}$ \\
\hline AZD1775 & $0.065 \mu \mathrm{M}$ & $0.026 \mu \mathrm{M}$ & $0.099 \mu \mathrm{M}$ \\
\hline MIDOSTAURIN & $0.062 \mu \mathrm{M}$ & $0.062 \mu \mathrm{M}$ & $0.087 \mu \mathrm{M}$ \\
\hline
\end{tabular}

Figure 6. Reduction of clonogenic growth of neoplastic MCs from two patients with MCL (MCL3 and MCL7; blue and green curves, respectively) and one patient with ASM (ASM18; red curve) as compared to two healthy donors (HDs; black and gray curves) in the presence of increasing doses of bortezomib (0.25-1 nM) (a), AZD1775+midostaurin combination (0.025-0.1 $\mu \mathrm{m})(\mathbf{b})$, AZD1775 alone (0.025-0.1 $\mu \mathrm{M})(\mathbf{c})$, midostaurin alone $(0.025-0.1 \mu \mathrm{M})(\mathbf{d})$. All the clonogenic survival rates are expressed as mean \pm s.d. of counts from three independent experiments. (e) Comparison of $\mathrm{LD}_{50}$ values for the different strategies.

Patients with evidence of $\mathrm{LOH}$ at 3p21.3 did not always harbor lower SETD2 protein levels as compared to patients with no $\mathrm{LOH}$. Thus, the biological significance of the recurrent $3 p 21.3 \mathrm{LOH}$ events that can be observed in SM remains, at present, unclear.

Ligand-independent activation of the KIT pathway, often resulting from the D816V KIT mutation, has a central role in SM pathogenesis. In acute myeloid leukemias, SETD2 has been found to be a critical cooperating event for a variety of major chromosomal or genetic aberrations that are known to be driver mutations in leukemogenesis. ${ }^{37}$ In these studies, SETD2 knockdown contributed to leukemia initiation, accelerated progression, enhanced the number and the self-renewal potential of leukemic stem cells and was associated with increased expression of mTOR and JAK-STAT pathway components-which are also important downstream effectors of KIT. It can be hypothesized that reduced SETD2 expression/absence and H3K36Me3 deficiency can cooperate with, and potentiate the effects of KIT constitutive activation to determine the phenotype of advanced SM. Increased DNA damage ${ }^{42}$ reported to occur in SETD2-deficient cells, would then be responsible for the accumulation of additional molecular/ cellular defects accounting for the phenotypic and clinical heterogeneity of the disease.

Our findings may have important therapeutic implications. Polychemotherapy, interferon, cladribine or midostaurin are currently the main options in advanced SM, and hematopoietic stem cell transplant is also considered in younger patients. ${ }^{43-45}$
However, treatment results are often unsatisfactory and many patients relapse on therapy or have resistant disease. In addition, in many cases with advanced SM-intensive therapy cannot be applied because of age or poor performance status. As a result, the prognosis of patients with advanced SM is poor and the median survival of patients with MCL is measurable in months. Our study points to bortezomib as a promising novel approach worth exploring clinically for the treatment of advanced SM. Bortezomib had already been reported to counteract the growth of cell lines and primary neoplastic MCs, an effect that had been attributed to the bortezomib-mediated re-expression of the proapoptotic factor BIM (suppressed in SM as a result of constitutive activation of the KIT/SCF pathway). ${ }^{46}$ However, the concentrations of bortezomib required to suppress proliferation and induce apoptosis were slightly lower than that required to re-express BIM, which had led to the hypothesis that bortezomib might act in part via additional mechanisms. We here show that bortezomib treatment at subnanomolar concentrations promotes SETD2 protein re-expression, reduces clonogenic potential and promotes apoptosis. Whether concomitant KIT D816V inhibition by midostaurin might exert an additive or synergistic effect was also tested-but the striking efficacy of bortezomib as single agent appeared not to be significantly enhanced further (Supplementary Figure 3). In renal cell carcinomas, it has been reported that SETD2-negative cells display elevated DNA damage ${ }^{42}$ and that SETD2 depletion abrogates p53 activation. ${ }^{47}$ It can be 
hypothesized that apoptosis triggered by bortezomib may at least in part result from restoration of a functional p53 checkpoint in a context of DNA damage.

We were also intrigued by the recent report suggesting that H3K36Me3-deficient tumors can be targeted in vitro and in vivo by AZD1775 (a Wee1 kinase inhibitor) through a mechanism of synthetic lethality ultimately resulting in dNTP starvation. ${ }^{34}$ AZD1775 alone or in combination with other targeted drugs or chemotherapy is currently being assessed in phase 2 studies for several types of solid tumors as well as for acute leukemias and myelodysplasias - which would greatly facilitate its repurposing. This prompted us to explore whether the elimination of H3K36Me3-deficient cells by AZD1775, alone or in combination with KIT D816V inhibition by midostaurin, might prove effective in vitro in our cell line model as well as in neoplastic MCs from patients with advanced SM. AZD1775 and midostaurin showed strong synergy in inducing apoptosis in HMC-1.1 cells, but only an additive effect in HMC-1.2 cells. Clonogenic growth of both cell line subclones was affected in a dose-dependent manner by AZD1775+midostaurin combination, that was slightly more effective than the single agents alone. In neoplastic MCs from patients with advanced SM, the combination of AZD1775 +midostaurin appeared to be slightly superior to the single agent strategy in two out of three cases, whereas in the remaining case AZD1775 as single agent showed the lowest LD $_{50}$. Additional testing in a greater number of patient samples will thus be required to better investigate the efficacy of a strategy using AZD1775 alone or in combination.

In conclusion, we show that non-genomic loss-of-function of SETD2 is a recurrent event in advanced SM. SETD2 and H3K36Me3 deficiency might cooperate with the ligand-independent activation of the KIT pathway to enhance leukemic transformation and progression and determine the phenotype of ASM and MCL. Targeting SETD2 down-modulation itself (with bortezomib or second-generation proteasome inhibitors) and/or SETD2/KIT cooperation are promising therapeutic strategies to improve the outcome of ASM and MCL patients.

\section{CONFLICT OF INTEREST}

The authors declare no conflict of interest.

\section{ACKNOWLEDGEMENTS}

This study was supported by NGS-PTL grant agreement: n. 306242 (GM), by AIRC IG grant 2015 (id.16996) (SS), and by Austrian Science Fund (FWF) grant F4704-B20 (PV).

\section{AUTHOR CONTRIBUTIONS}

Contribution: SS and GM designed the study, supervised the study, analyzed and interpreted results. MMancini, CDB, MR, MManfrini, MMeggendorfer, RC, VG, MCF, AP, LB, EZ, MD, LR, FM and TH performed experiments, analyzed and interpreted results. CP, LP, RZ, LS, GS, FA, SM, CE, PS, DG, PT, FC, GP and PV provided patient samples and clinical data. $M C$ coordinated the clinical and research activities. All authors contributed to drafting the manuscript and gave final approval for submission.

\section{REFERENCES}

1 Theoharides TC, Valent P, Akin C. Mast cells, mastocytosis, and related disorders. N Engl J Med 2015; 373: 163-172.

2 Valent P, Horny HP, Escribano L, Longley BJ, Li CY, Schwartz LB et al. Diagnostic criteria and classification of mastocytosis: a consensus proposal. Leuk Res 2001; 25: 603-625.

3 Horny HP, Akin C, Metcalfe DD, Escribano L, Bennett CB, Valent P et al. Mastocytosis (Mast cell disease). In: Swerdlow SH, Campo E, Harris NL, Jaffe ES, Pileri SA, Stein $\mathrm{H}$, et al. (eds). World Health Organization (WHO) Classification of Tumours. Pathology and Genetics. Tumours of Haematopoietic and Lymphoid Tissues. IARC Press: Lyon, 2008, pp 54-63.
4 Arber DA, Orazi A, Hasserjian R, Thiele J, Borowitz MJ, Le Beau MM et al. The 2016 revision to the World Health Organization classification of myeloid neoplasms and acute leukemia. Blood 2016; 127: 2391-2405.

5 Kitamura Y, Tsujimura T, Jippo T, Kasugai T, Kanakura Y. Regulation of development, survival and neoplastic growth of mast cells through the c-kit receptor. Int Arch Allergy Immunol 1995; 107: 54-56.

6 Arock M, Sotlar K, Akin C, Broesby-Olsen S, Hoermann G, Escribano L et al. KIT mutation analysis in mast cell neoplasms: recommendations of the European Competence Network on Mastocytosis. Leukemia 2015; 29: 1223-1232.

7 Zappulla JP, Dubreuil P, Desbois S, Letard S, Hamouda NB, Daeron M et al. Mastocytosis in mice expressing human Kit receptor with the activating Asp816Val mutation. J Exp Med 2005; 202: 1635-1641.

8 Mayerhofer M, Gleixner KV, Hoelbl A, Florian S, Hoermann G, Aichberger KJ et al. Unique effects of KIT D816V in BaF3 cells: induction of cluster formation, histamine synthesis, and early mast cell differentiation antigens. J Immunol 2008; 180: 5466-5476.

9 Tefferi A, Levine RL, Lim KH, Abdel-Wahab O, Lasho TL, Patel J et al. Frequent TET2 mutations in systemic mastocytosis: clinical, KITD816V and FIP1L1-PDGFRA correlates. Leukemia 2009; 23: 900-904.

10 Tefferi A. Novel mutations and their functional and clinical relevance in myeloproliferative neoplasms: JAK2, MPL, TET2, ASXL1, CBL, IDH and IKZF1. Leukemia 2010; 24: 1128-1138.

11 Schwaab J, Schnittger S, Sotlar K, Walz C, Fabarius A, Pfirrmann M et al. Comprehensive mutational profiling in advanced systemic mastocytosis. Blood 2013; 122: $2460-2466$.

12 Jawhar M, Schwaab J, Schnittger S, Sotlar K, Horny HP, Metzgeroth G et al. Molecular profiling of myeloid progenitor cells in multi-mutated advanced systemic mastocytosis identifies KIT D816V as a distinct and late event. Leukemia 2015; 29: 1115-1122.

13 Jawhar M, Schwaab J, Schnittger S, Meggendorfer M, Pfirrmann M, Sotlar K et al. Additional mutations in SRSF2, ASXL1 and/or RUNX1 identify a high-risk group of patients with KIT D816V(+) advanced systemic mastocytosis. Leukemia 2016; 30: 136-143.

14 Jawhar M, Schwaab J, Hausmann D, Clemens J, Naumann N, Henzler T et al. Splenomegaly, elevated alkaline phosphatase and mutations in the SRSF2/ASXL1/ RUNX1 gene panel are strong adverse prognostic markers in patients with systemic mastocytosis. Leukemia 2016; 30: 2342-2350.

15 Pardanani A, Lasho T, Barraco D, Patnaik M, Elala Y, Tefferi A. Next generation sequencing of myeloid neoplasms with eosinophilia harboring the FIP1L1PDGFRA mutation. Am J Hematol 2016; 91: E10-E11.

16 Spector MS, lossifov I, Kritharis A, He C, Kolitz JE, Lowe SW et al. Mast-cell leukemia exome sequencing reveals a mutation in the lgE mast-cell receptor beta chain and KIT V654A. Leukemia 2012; 26: 1422-1425.

17 Sundstrom M, Vliagoftis $H$, Karlberg P, Butterfield JH, Nilsson K, Metcalfe DD et al. Functional and phenotypic studies of two variants of a human mast cell line with a distinct set of mutations in the c-kit proto-oncogene. Immunology 2003; 108: 89-97.

18 Kanakura Y, Furitsu T, Tsujimura T, Butterfield JH, Ashman LK, Ikeda $\mathrm{H}$ et al. Activating mutations of the c-kit proto-oncogene in a human mast cell leukemia cell line. Leukemia 1994; 8: S18-S22.

19 Chou TC. Drug combination studies and their synergy quantification using the Chou-Talalay method. Cancer Res 2010; 70: 440-446.

20 Team RC. R: a language and environment for statistical computing. R Foundation for Satistical Computing: Vienna, Austria, 2016.

21 Kudithipudi S, Jeltsch A. Role of somatic cancer mutations in human protein lysine methyltransferases. Biochim Biophys Acta 2014; 1846: 366-379.

22 Chopra M, Bohlander SK. Disturbing the histone code in leukemia: translocations and mutations affecting histone methyl transferases. Cancer Genet 2015; 208: 192-205.

23 Edmunds JW, Mahadevan LC, Clayton AL. Dynamic histone H3 methylation during gene induction: HYPB/Setd2 mediates all H3K36 trimethylation. EMBO J 2008; 27: 406-420.

24 Kizer KO, Phatnani HP, Shibata Y, Hall H, Greenleaf AL, Strahl BD. A novel domain in Set2 mediates RNA polymerase II interaction and couples histone H3 K36 methylation with transcript elongation. Mol Cell Biol 2005; 25: 3305-3316.

25 Li B, Pattenden SG, Lee D, Gutierrez J, Chen J, Seidel C et al. Preferential occupancy of histone variant $\mathrm{H} 2 \mathrm{AZ}$ at inactive promoters influences local histone modifications and chromatin remodeling. Proc Natl Acad Sci USA 2005; 102: 18385-18390.

26 Vojnic E, Simon B, Strahl BD, Sattler M, Cramer P. Structure and carboxyl-terminal domain (CTD) binding of the Set2 SRI domain that couples histone H3 Lys36 methylation to transcription. J Biol Chem 2006; 281: 13-15.

27 Yuan W, Xie J, Long C, Erdjument-Bromage H, Ding X, Zheng $Y$ et al. Heterogeneous nuclear ribonucleoprotein $L$ Is a subunit of human KMT3a/Set2 complex required for H3 Lys-36 trimethylation activity in vivo. J Biol Chem 2009; 284: 15701-15707. 
28 Hesson LB, Cooper WN, Latif F. Evaluation of the 3p21.3 tumour-suppressor gene cluster. Oncogene 2007; 26: 7283-7301.

29 Genovese G, Kahler AK, Handsaker RE, Lindberg J, Rose SA, Bakhoum SF et al. Clonal hematopoiesis and blood-cancer risk inferred from blood DNA sequence. N Engl J Med 2014; 371: 2477-2487.

30 Jaiswal S, Fontanillas P, Flannick J, Manning A, Grauman PV, Mar BG et al. Agerelated clonal hematopoiesis associated with adverse outcomes. $N$ Engl J Med 2014; 371: 2488-2498.

31 Xie M, Lu C, Wang J, McLellan MD, Johnson KJ, Wendl MC et al. Age-related mutations associated with clonal hematopoietic expansion and malignancies. Nat Med 2014; 20: 1472-1478.

32 Gotlib J, Berube C, Growney JD, Chen CC, George TI, Williams C et al. Activity of the tyrosine kinase inhibitor PKC412 in a patient with mast cell leukemia with the D816V KIT mutation. Blood 2005; 106: 2865-2870.

33 Gleixner KV, Mayerhofer M, Aichberger KJ, Derdak S, Sonneck K, Bohm A et al. PKC412 inhibits in vitro growth of neoplastic human mast cells expressing the D816V-mutated variant of KIT: comparison with AMN107, imatinib, and cladribine (2CdA) and evaluation of cooperative drug effects. Blood 2006; 107: 752-759.

34 Pfister SX, Markkanen E, Jiang Y, Sarkar S, Woodcock M, Orlando G et al. Inhibiting WEE1 selectively kills histone H3K36me3-deficient cancers by dNTP starvation. Cancer Cell 2015; 28: 557-568.

35 Dalgliesh GL, Furge K, Greenman C, Chen L, Bignell G, Butler A et al. Systematic sequencing of renal carcinoma reveals inactivation of histone modifying genes. Nature 2010; 463: 360-363.

36 Li J, Duns G, Westers H, Sijmons R, van den Berg A, Kok K. SETD2: an epigenetic modifier with tumor suppressor functionality. Oncotarget 2016; 7: 50719-50734.

37 Zhu X, He F, Zeng H, Ling S, Chen A, Wang Y et al. Identification of functional cooperative mutations of SETD2 in human acute leukemia. Nat Genet 2014; 46: 287-293.

38 Mar BG, Bullinger LB, McLean KM, Grauman PV, Harris MH, Stevenson $\mathrm{K}$ et al. Mutations in epigenetic regulators including SETD2 are gained during relapse in paediatric acute lymphoblastic leukaemia. Nat Commun 2014; 5: 3469.

39 Liu W, Fu Q, An H, Chang Y, Zhang W, Zhu Y et al. Decreased expression of SETD2 predicts unfavorable prognosis in patients with nonmetastatic clear-cell renal cell carcinoma. Medicine (Baltimore) 2015; 94: e2004.
40 Wang J, Liu L, Qu Y, Xi W, Xia Y, Bai Q et al. Prognostic value of SETD2 expression in patients with metastatic renal cell carcinoma treated with tyrosine kinase inhibitors. J Urol 2016; 196: 1363-1370.

41 Parker H, Rose-Zerilli MJ, Larrayoz M, Clifford R, Edelmann J, Blakemore S et al. Genomic disruption of the histone methyltransferase SETD2 in chronic lymphocytic leukaemia. Leukemia 2016; 30: 2179-2186.

42 Kanu N, Gronroos E, Martinez P, Burrell RA, Yi Goh X, Bartkova J et al. SETD2 lossof-function promotes renal cancer branched evolution through replication stress and impaired DNA repair. Oncogene 2015; 34: 5699-5708.

43 Valent P. Diagnosis and management of mastocytosis: an emerging challenge in applied hematology. Hematology Am Soc Hematol Educ Program 2015; 2015: 98-105.

44 Ustun C, Reiter A, Scott BL, Nakamura R, Damaj G, Kreil S et al. Hematopoietic stem-cell transplantation for advanced systemic mastocytosis. J Clin Oncol 2014; 32: 3264-3274.

45 Barete S, Lortholary O, Damaj G, Hirsch I, Chandesris MO, Elie C et al. Long-term efficacy and safety of cladribine (2-CdA) in adult patients with mastocytosis. Blood 2015; 126: 1009-1016, quiz 1050.

46 Aichberger KJ, Gleixner KV, Mirkina I, Cerny-Reiterer S, Peter B, Ferenc V et al. Identification of proapoptotic Bim as a tumor suppressor in neoplastic mast cells: role of KIT D816V and effects of various targeted drugs. Blood 2009; 114: 5342-5351.

47 Carvalho S, Vitor AC, Sridhara SC, Martins FB, Raposo AC, Desterro JM et al. SETD2 is required for DNA double-strand break repair and activation of the p53mediated checkpoint. Elife 2014; 3: e02482.

(2)(2) This work is licensed under a Creative Commons Attribution(c) NC SA NonCommercial-ShareAlike 4.0 International License. The images or other third party material in this article are included in the article's Creative Commons license, unless indicated otherwise in the credit line; if the material is not included under the Creative Commons license, users will need to obtain permission from the license holder to reproduce the material. To view a copy of this license, visit http:// creativecommons.org/licenses/by-nc-sa/4.0/

(c) The Author(s) 2018

Supplementary Information accompanies this paper on the Leukemia website (http://www.nature.com/leu) 